\title{
Y a-t-il un effet de taille et d'encastrement pour les fondations superficielles dans les sables?
}

J.L. BRIAUD

Professor and Holder of the Spencer

J. Buchanan Chair

Dpt. of Civil Engineering

Texas A\&M University

College Station

Texas 77843-3136

Etats-Unis

briaud@tamu.edu

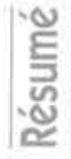

L'effet de la taille d'une fondation superficielle et l'effet de son encastrement sur la courbe donnant le déplacement en fonction de la charge sont l'objet de cet article. Des arguments théoriques et surtout des données expérimentales sont présentés. Ils indiquent la chose suivante. Si le comportement de la fondation est représenté par la courbe donnant la pression sous la fondation divisée par une mesure de la résistance du sol dans la zone influencée par la fondation en fonction du tassement divisé par la largeur de la fondation, toutes les courbes ainsi obtenues pour des fondations de taille et d'encastrement différents se regroupent dans une bande serrée pour un sable donné. L'existence de cette bande serrée est en accord avec la méthode de la courbe charge-tassement proposée par Briaud et Jeanjean (1994).

Mots-clés : fondations superficielles, sable, essaí de chargement, courbe charge-déplacement, effet de taille, effet d'encastrement.

\section{Is there a scale and embedment effect for shallow foundations in sand?}

arguments and several load tests results are presented. They show the following. If the behavior of the shallow foundation is represented by the curve giving the average pressure under the foundation divided by a measure of the soil résistance within the foundation zone of influence as a function of the settlement divided by the foundation width, all the curves obtained for different foundation sizes and different foundation embedments collapse into a narrow band for a given sand. The existence of such a narrow band is consistent with the load settlement curve method proposed by Briaud and Jeanjearn (1994).

Key words: shallow foundations, sand, load test, load-settlement curve, scale effect, embedment effect. 


\section{1}

\section{Introduction}

En 1994, à l'occasion de la conférence de l'ASCE intitulée « Settlement '94), Briaud et Jeanjean (1994) ont présenté les résultats d'essais de chargement sur cing fondations superficielles reposant sur le sable du Site national de recherche en gêotechnique à Texas A\&M University. Ces fondations carrées de $1 \mathrm{~m} \times 1 \mathrm{~m}$. $1.5 \mathrm{~m} \times 1.5 \mathrm{~m}, 2.5 \mathrm{~m} \times 2.5 \mathrm{~m}, 3 \mathrm{~m} \times 3 \mathrm{~m}$, et $3 \mathrm{~m} \times 3 \mathrm{~m}$ étaient encastrées de $0,75 \mathrm{~m}$ au-dessous du niveau du sol naturel. Les résultats de ces essais subventionnés par la FHWA se trouvent dans les publications de Briaud et Gibbens (1994, 1997, 1999). Briaud et Jeanjean ont montré que lorsque les résultats de ces cing essais de chargement sont présentés sous la forme de pression movenne sous la fondation en fonction du tassement divisé par la largeur de la fondation, les cinq courbes ainsi obtenues se regroupent dans une bande très serrée. Ils concluent qu'il n'y a pas d'effet de taille pour ces fondations et proposent une méthode pour prévoir la courbe charge-déplacement pour une fondation superficielle reposant dans du sable. L'absence d'effet de taille fut l'objet d'un débat lors de la conférence. Un éminent ingénieur suggéra que l'absence apparente d'effet de taille était due à l'annulation de l'augmentation de pression due à l'augmentation de taille par la diminution d'encastrement relatif. Cet article est le résultat d'efforts supplémentaires pour élucider la question de l'effet de taille et d'encastrement sur le comportement des fondations superficielles dans les sables.

Ce sujet a été étudié par beaucoup de chercheurs en commençant par Taylor en 1948. Ils ont tous proposé des explications variées et plus ou moins compliquées pour le fait que la théorie ne correspond pas complètement à la réalité particulièrement en ce qui concerne la capacité portante ultime. On note les explications liées à la non-linéarité de l'enveloppe de rupture (DeBeer, 1965: Ovesen, 1975; Graham et Hovan, 1986; Hettler et Gudehus, 1988; Kutter et al. 1988; Bolton et Lau, 1989; Shiraishi, 1990; Perkins, 1995) liées à un mécanisme de rupture progressive (Muhs, 1963; Yamacruchi et al. 1976), liées à la dilatance des sols (Bolton, 1986), liées au rapport de la taille de la fondation à la taille des grains (Steenfelt, 1977), liées à plusieurs facteurs (Corte. 1980: Habib, 1985; Kimura et al., 1985; Garnier, 1997; Perkins et Madson, 2000). Dans cet article, on présente une raison différente et très simple pour expliquer l'écart entre les observations expérimentales et la théorie.

\section{2}

\section{L'équation de capacité portante ultime}

L'équation de capacité portante ultime pour une semelle filante dans un sol de caractéristiques c' et $\phi^{\prime}$ uniforme s'exprime (Terzaghi et al., 1996):

$$
\mathrm{q}_{\mathrm{u}}=\mathrm{c}^{\prime} \mathrm{N}_{\mathrm{c}}+0,5 \gamma \mathrm{BN}_{\mathrm{u}}+\gamma \mathrm{DN}
$$

où $\mathrm{q}_{\text {in }}$ est la capacité portante ultime, $\mathrm{c}^{\mathrm{s}}$ est la cohésion effective, $\gamma$ le poids volumique effectif, B la largeur de la fondation. D l'encastrement, et $N_{,} N_{,} N_{c}$ sont les facteurs de capacité portante qui dépendent de l'angle de frottement effectif $\phi^{\prime}$. Pour un sable sans cohésion et une semelle filante sans encastrement, l'équation (1) indique que la capacité portante ultime augmente linéairement avec la largeur de la fondation. De même, cette équation indique que la capacité portante ultime augmente linéairement avec l'encastrement.

Trois des hypothèses de l'équation (1) sont que le sable est sec, qu'il a une valeur de $\phi^{\prime}$ constante et une valeur de $\gamma$ constante. Le fait que le sable est sec et que $\gamma$ est constant conduit à une contrainte effective $\sigma$ qui augmente linéairement avec la profondeur. La résistance au cisaillement $\tau$, augmente donc avec la profondeur (Fig. 1a) puisqu'elle s'écrit:

$$
\tau_{f}=\sigma \tan \phi
$$

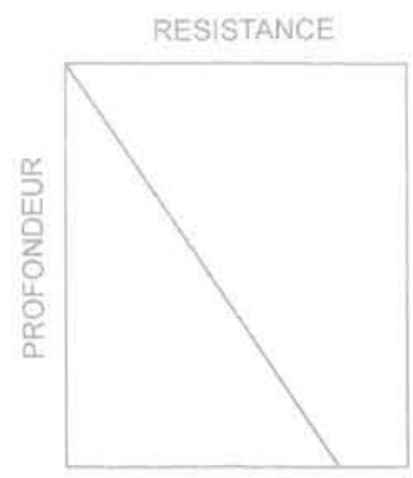

(a)

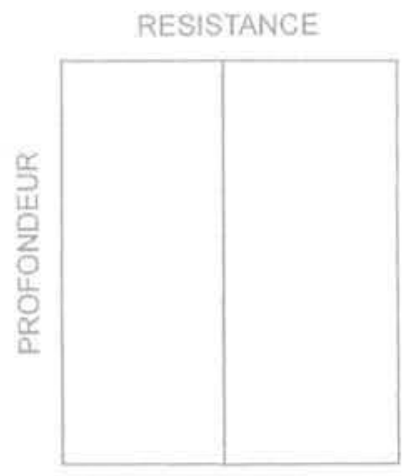

(b)
96.1 Deux types de profil de résistance: (a) augmentation linéaire, (b) constant. Two types of resistance profiles: (a) linear increase, (b) constant.

On peut donc s'attendre à ce que l'équation (1) donne de bonnes prévisions quand la résistance du sol augmente linéairement en fonction de la profondeur. Un profil de résistance au SPT, au CPT, ou un profil de pression limite au PMT peuvent indiquer si cette augmentation linéaire en fonction de la profondeur est légitime ou non pour un site donné. Bien souvent près de la surface, où sont fondées les fondations superficielles, les sols ne sont pas saturés et on a affaire à des profils où la résistance est pratiquement constante en fonction de la profondeur. Dans ce cas (Fig. 1b) on peut s'attendre à des difficultés de prévision quand on utilise l'équation (1) car les hypothèses ne correspondent pas à la réalité. On pourrait modifier l'équation (1) comme suit:

$$
q_{\mathrm{u}}=\sigma_{\mathrm{b}} \mathrm{N}_{y}
$$

où $\sigma_{b}$ est la contrainte effective moyenne sous la fondation; ou même

$$
\mathrm{q}_{\mathrm{u}}=\mathrm{r} \mathrm{k}
$$

où $r$ est une mesure de la résistance du sol $\left(q, p_{1}\right.$, N) et k est un coefficient de capacité portante. C'est la solution adoptée dans le cas de calcul direct à partir des essais in situ. Dans ce sens, cette approche est supérieure à l'approche basée sur l'équation (1). Dans le cas de résistance constante, on peut donc aussi s'attendre à ce que la capacité portante n'augmente pas avec la grandeur de la fondation puisque la résistance du sol dans la zone mobilisée par la fondation reste constante. Cette discussion montre bien que le profil de résistance du sol a un impact direct sur l'effet de taille. On imagine de même que ce profil aura un impact semblable sur l'effet d'encastrement. 


\section{L'équation d'élasticité}

La théorie de l'élasticité est souvent utilisée pour calculer le tassement d'une fondation superficielle reposant sur du sable. L'équation (e.g. Mayne et Poulos, 1999)

$$
\mathrm{s}=(\mathrm{qB} / \mathrm{E})\left(1-v^{2}\right) I_{\mathrm{G}} I_{F} I_{E}
$$

donne le tassement $\mathrm{s}$ en fonction de la pression $\mathrm{q}$, du diamètre $B$ de la fondation circulaire, du module $\mathrm{d}^{\prime}$ Young E, et du coefficient de Poisson $v$, ainsi que des facteurs $I_{G}, I_{F}$, et $I_{E}$ caractérisant l'augmentation du module en fonction de la profoncleur, de la flexibilité de la fondation, et de l'encastrement. Les abaques présentées par Mayne et Poulos donnant les valeurs de $I_{C}$ et $I_{E}$ sont présentées sur les figures 2 et 3 . Pour une fondation rigide circulaire à la surface d'un sol ayant un module constant avec la profondeur, l'équation (5) devient:

$$
\mathrm{s}=(\mathrm{qB} / \mathrm{E})\left(1-v^{2}\right) \pi / 4
$$

Cette équation montre bien que la courbe qui représente la variation de q en fonction de $\mathrm{s} / \mathrm{B}$ sera indépendante de la grandeur de la fondation et ne dépendra que des propriétés du sol. II n'y aura pas d'effet de taille pour cette courbe. D'un autre côté, le facteur I $I_{E}$ (Fig. 3) indique que l'augmentation de l'encastrement d'une fondation de largeur donnée dans un sol au module constant, réduit les tassements. La réduction maximale est de 10 à $15 \%$ pour des valeurs raisonnables du coefficient de Poisson $(0,3$ à 0,5$)$ et pour des encastrements habituels ( $\mathrm{D} / \mathrm{B}$ de 0 à 2 ).

Si on considère maintenant un sol où le module

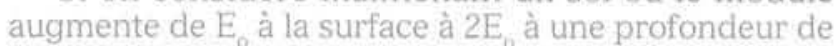
$2 \mathrm{~B}$ sous une fondation rigide, le coefficient $\beta$ (Fig. 2) est alors égal à 2 et $I_{G}$ à 0,59 . Si on double la largeur de la fondation sur ce même profil de module, le coefficient $\beta$ est égal à 1 et $\mathrm{I}_{\mathrm{g}}$ vaut 0,5 . L'effet de taille dans ce cas est considéré comme étant donné par le rapport des valeurs de s/B pour les deux fondations superficielles soumises à la même pression q. Ce rapport se réduit au rapport des valeurs de $l_{G}$ (équation 5 ) soit 0,85 ou une différence de $15 \%$.

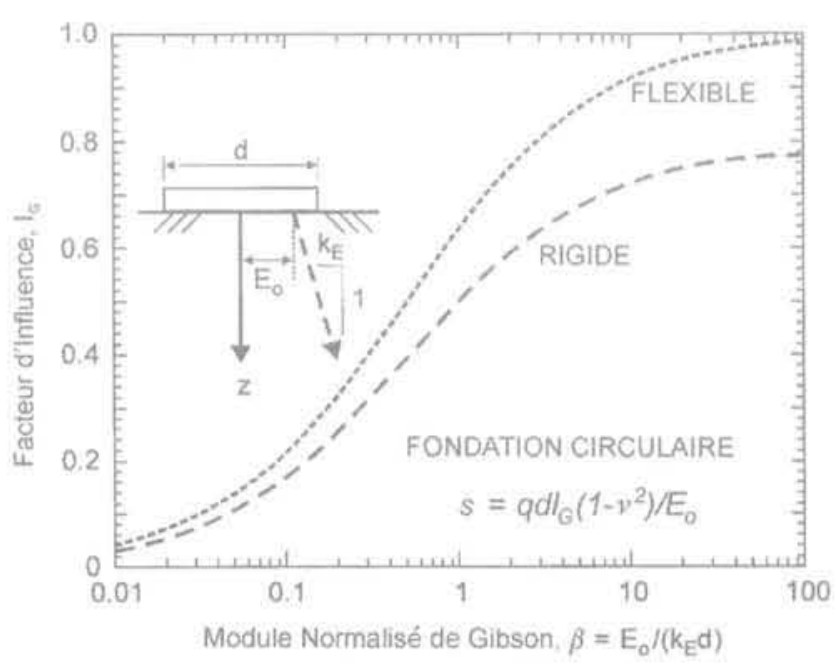

FIG.2 Facteur $I_{6}$ pour l'influence du profil de module (d'après Mayne et Poulos, 1999). Influence factor $I_{c}$ for the modulus profile (after Mayne and Poulos, 1999).

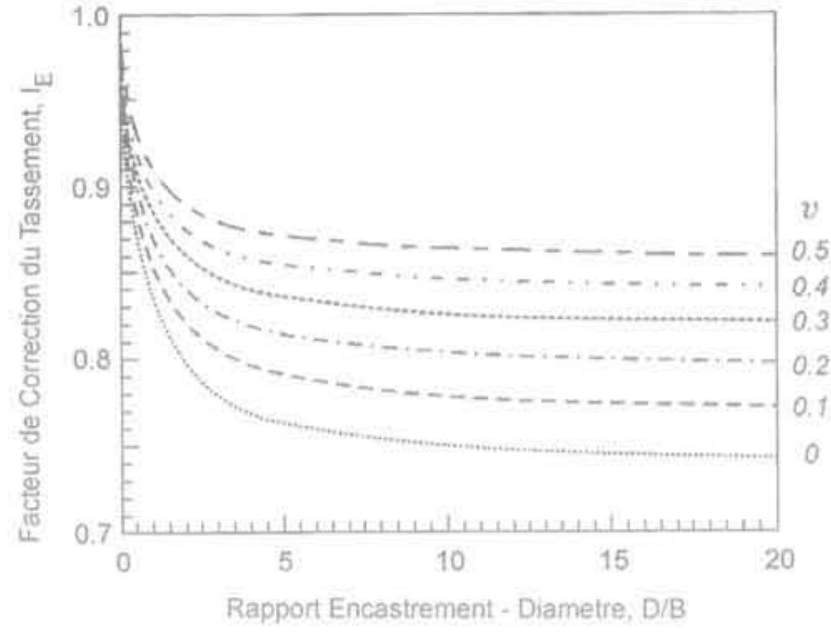

FIG.3 Facteur d'encastrement $\mathrm{I}_{\mathrm{F}}$ (d'après Mayne et Poulos, 1999).

Influence factor If $_{\text {for }}$ embedment depth (after Mayne and Poulos, 1999).

Cette discussion sur le tassement élastique montre bien que la variation du module avec la profondeur a un impact direct sur les effets de taille et d'encastrement. De plus, elle montre que pour des conditions moyennes, cet impact est minime $(<15 \%)$. Puisque d'après la théorie, le profil de résistance (capacité portante ultime) et le profil de module (tassement) ont un impact sur les effets de taille et d'encastrement, on s'est efforcé d'accumuler des résultats expérimentaux d'essais de fondation pour élucider cette influence.

\section{4}

\section{Les essais sur grandes fondations de Texas A\&M University}

Une série d'essais de chargement sur des grandes fondations superficielles a été effectuée en 1993 sur le Site national de recherche expérimentale en géotechnique à Texas A\&M University (NGES-TAMU). Les résultats dé ces essais ont déjà été publiés (Briaud, Gibbens, 1999); on les résume ici en soulignant les observations sur l'effet de taille, Cinq fondations superficielles en béton ont été construites sur le site NGES-TAMU; leurs dimensions étaient de $1 \mathrm{~m} \times 1 \mathrm{~m}$, $1,5 \mathrm{~m} \times 1,5 \mathrm{~m}, 2,5 \mathrm{~m} \times 2,5 \mathrm{~m}, 3 \mathrm{~m} \times 3 \mathrm{~m}$ et $3 \mathrm{~m} \times 3 \mathrm{~m}$. Toutes ces fondations faisaient 1,5 m d'épaisseur et étaient encastrées de $0,75 \mathrm{~m}$ dans le sable. Le sable est un sable silteux à densité relative moyenne. Le profil de résistance de ce sable est illustré par le graphique indiquant le nombre de coup de battage $\mathrm{N}$ au SPT en fonction de la profondeur (Fig. 4). On note que ce graphique indique une résistance pratiquement constante avec la profondeur. La nappe phréatique est à $4,9 \mathrm{~m}$ de profondeur.

Les résultats des essais sont représentés par la figure 5 donnant la pression en fonction du tassement divisé par la largeur de la fondation. Comme on le voit, les cinq courbes se regroupent dans une bande étroite et l'effet de taille est négligeable. La validité de cette conclusion est affaiblie par le fait que ces fondations n'ont pas le même encastrement relatif. Si on ignore l'effet possible des différents encastrements relatifs, 
NOMBRE DE COUP, N (coup/0.3m)

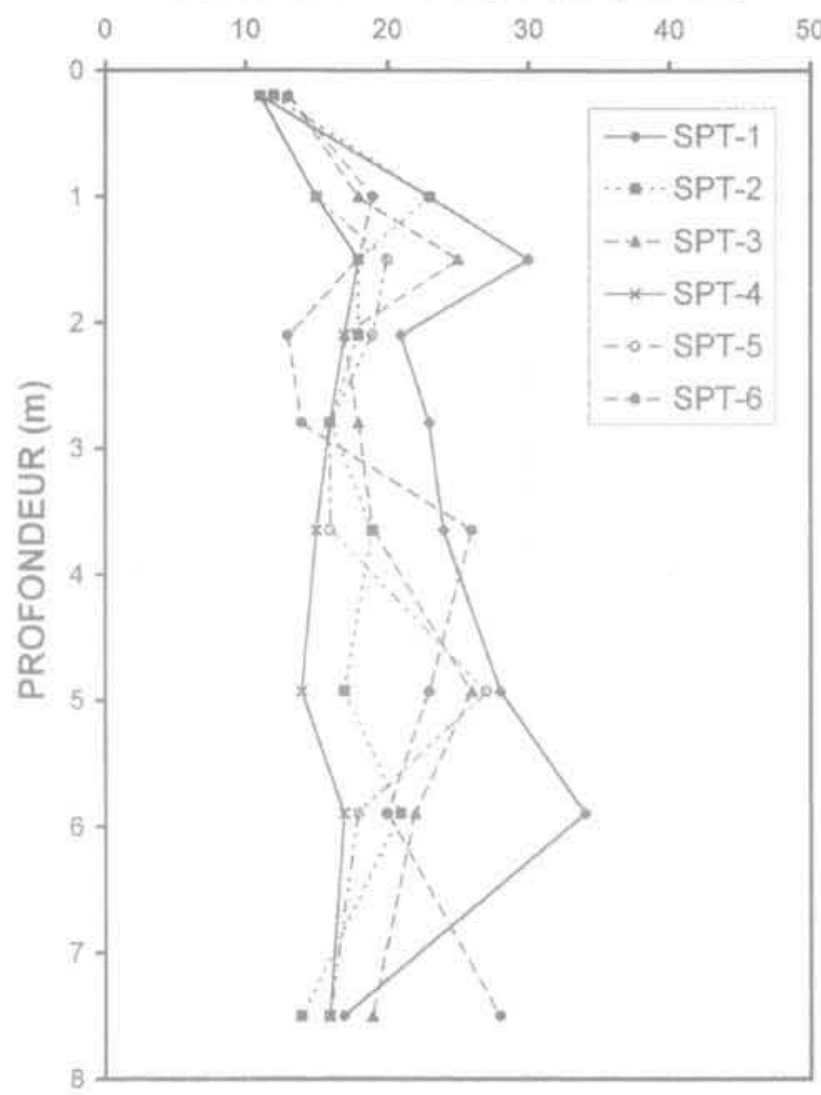

FIG. 4 Profil SPT pour le site de Texas A\&M University.

SPT profile for the Texas A\&M University site.

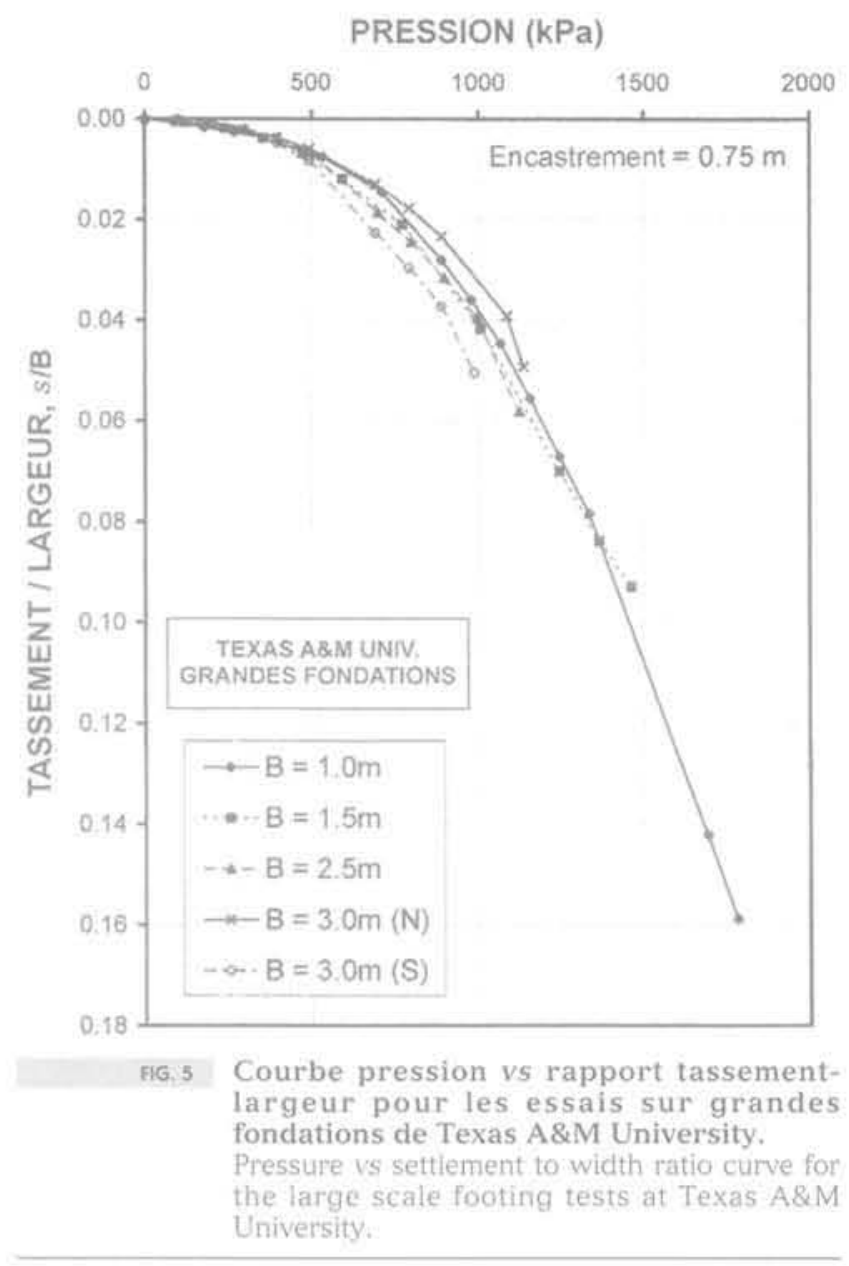

d'environ $2 \mathrm{~mm} / \mathrm{min}$ jusqu'à un enfoncement égal au $1 / 10$ de la larqeur de la plaque. A ce moment, la vitesse de pénétration a êté augmentée à $1 \mathrm{~mm} / \mathrm{s}$ et la plaque a étê enfoncée d'un mètre dans le sable où, le cas échéant, jusqu'à ce qu'on atteigne la réaction maximale du camion $(\sim 190 \mathrm{kN})$. La force appliquée a été mesurée avec la cellule de mesure de force du pénétromètre et le tassement de la plaque avec le système de câble donnant la mesure de déplacement du camion CPT. Ce système de mesure de déplacement, qui d'habitude se trouve à l'intérieur du camion de mesure, a été sorti du camion, une extrémité du câble a été montée directement sur la poutre de référence pour le tassement, et l'autre extrémité a été attachée sur la plaque. Un exemple des résultats est donné sur la figure 7 pour la plaque de $0,3 \mathrm{~m}$ de côté encastrée à $0,6 \mathrm{~m}$ de profondeur. Les résultats complets sont donnés par Barfknecht et Briaud (1999).

La pression correspondant à un tassement égal au $1 / 10$ de la largeur de la plaque est notée $p_{01}$. La pression correspondant à un tassement égal au 1/100 de la largeur de la plaque est notée $\mathrm{p}_{001}$. La résistance de pointe moyenne dans la zone d'influence de la plaque, évaluée à une profondeur égale à la largeur de la plaque, est appelée q. Tous les résultats des essais de plaque sont présentés sur deux figures: $p_{01} / q_{i u}$ fonction de $\mathrm{D} / \mathrm{B}$ est sur la figure 8 et $\mathrm{p}_{\mathrm{og}} / \mathrm{q}_{\mathrm{ct}}$ fonction de $\mathrm{D} / \mathrm{B}$ sur la figure 9. La normalisation de $p_{0.1}$ et $p_{0,01}$ par $q_{c a}$ du sondage CPT le plus proche de l'essai de plaque a été rendue nécessaire par l'hétérogénéité des $0,5 \mathrm{~m}$ de sable en surface. En effet les résultats des sondages CPT sur la figure 6 démontrent que cette hétérogénéité d'une tige de péétromètre et un camion CPT a été utilisé pour pousser la plaque dans le sable à une vitesse 


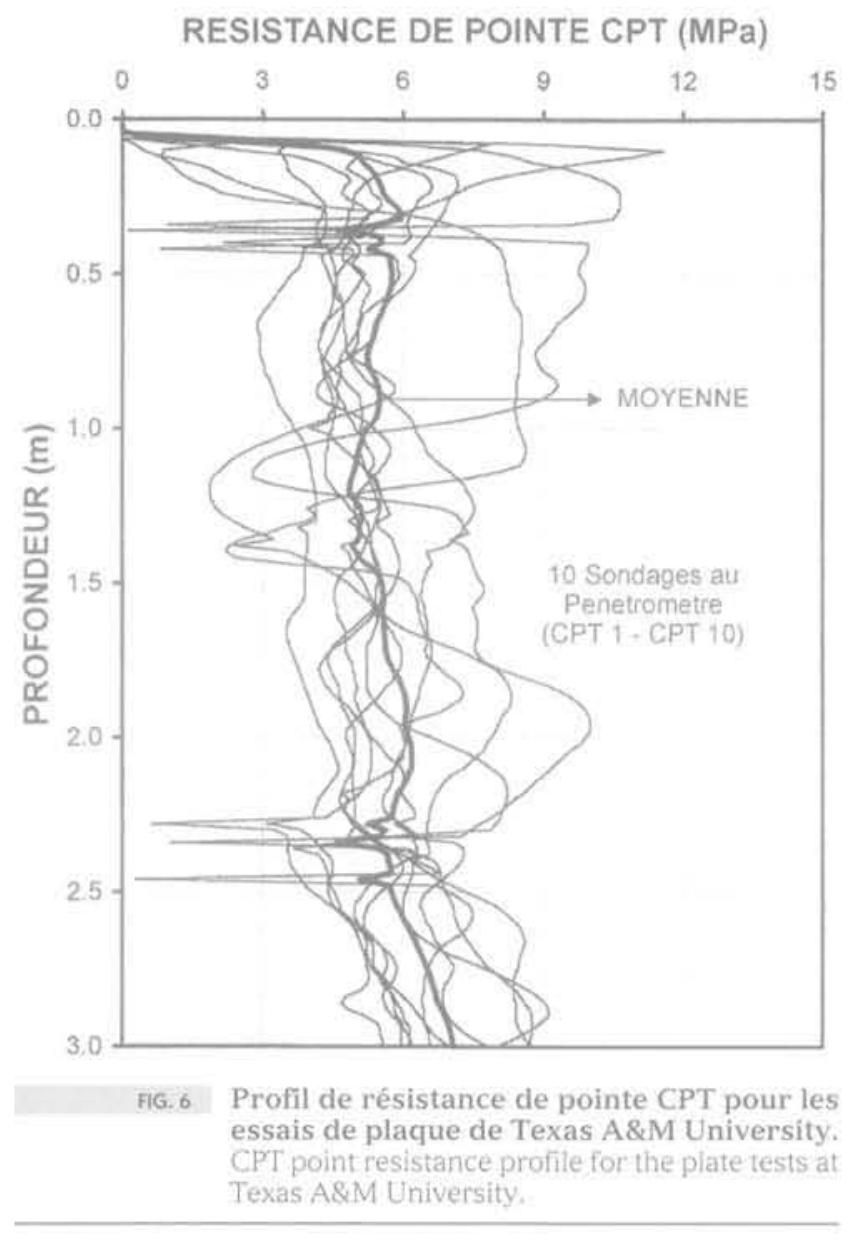

existe en surface et diminue avec la profondeur. Les figures 8 et 9 indiquent que, en dépit de cette hétérogénéité, les rapports $p_{01} / q_{\text {in }}$ et $p_{001} / q_{1}$ sont regroupés dans des plages de valeurs assez étroites et sont indépendants de la dimension et de l'encastrement de la plaque. La variation de ces rapports est plus importante en surface oủ l'hétérogénéité est elle aussi plus importante.

D'autres essais de fondation et de plaque trouvés dans les publications récentes ont été rassemblés pour pouvoir confirmer ou contredire l'absence d'effet de taille et d'encastrement sur les courbes d'enfoncement normalisées observées sur les essais de Texas A\&M University.

\section{6}

\section{Les essais de fondation d'Ismael (1985)}

Ismael (1985) a conduit une campagne d'essais d'enfoncement sur des petites fondations superficielles en béton reposant sur du sable au Koweit. Les fondations étaient carrées et avaient pour grandeur: $0,25 \mathrm{~m} \times$ $0,25 \mathrm{~m}, 0,5 \mathrm{~m} \times 0,5 \mathrm{~m}, 0,75 \mathrm{~m} \times 0,75 \mathrm{~m}$ et $1 \mathrm{~m} \times 1 \mathrm{~m}$. L'encastrement a varié de $0,5 \mathrm{~m}, 1 \mathrm{~m}, 1,5 \mathrm{~m}$ à $2 \mathrm{~m}$ dans un sable à granulométrie bien étalée (SW) et à densité relative moyenne, avec un peu de silt jusqu'à une profondeur de $4 \mathrm{~m}$. Le nombre moyen de coup de battage $\mathrm{N}$ au SPT est de 20 coups $/ 0,3 \mathrm{~m}$ et le profil est relativement constant avec la profondeur (Ismael, 1999, communication par mail). La nappe est à $2,8 \mathrm{~m}$ de profondeur.

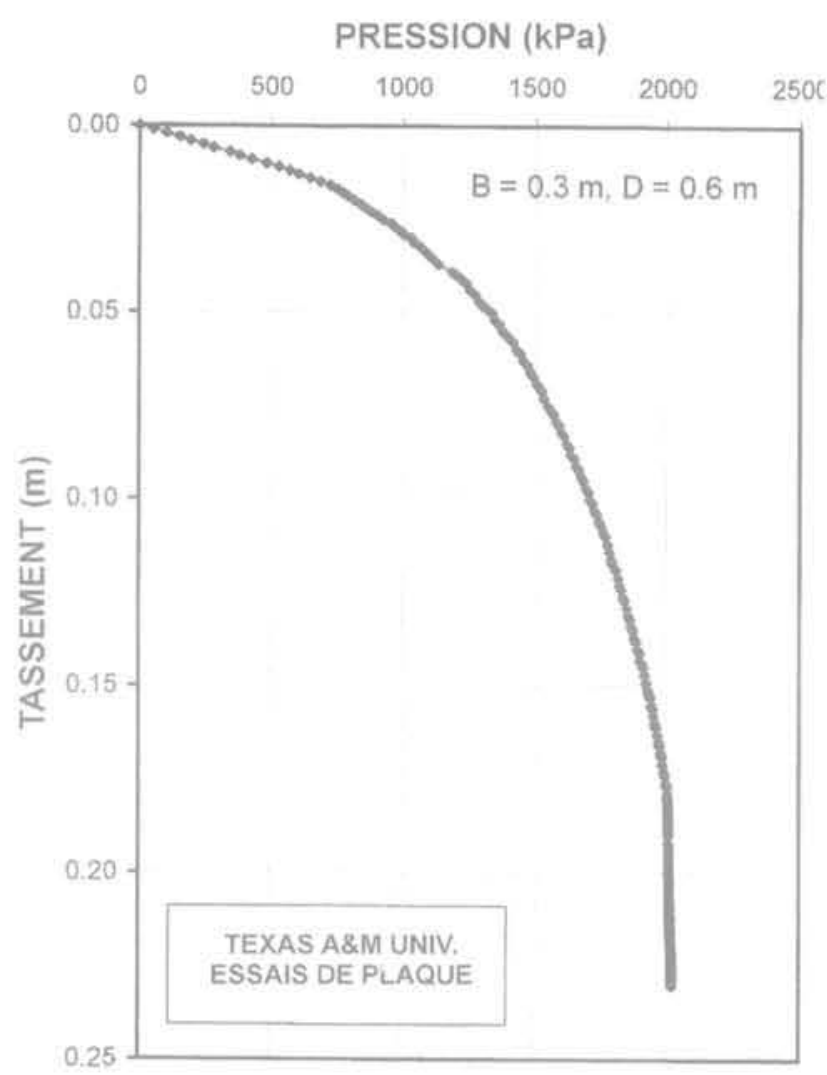

FG. 7 Courbe pression vS, tassement pour une plaque carrée de $0,3 \mathrm{~m}$ de côté et un encastrement de $0,6 \mathrm{~m}$ au site de sable de Texas A\&M University.

Pressure vs. Settlement curve for a $0.3 \mathrm{~m}$ by $0.3 \mathrm{~m}$ square plate embedded $0.6 \mathrm{~m}$ at the sand site of Texas A\&M University.

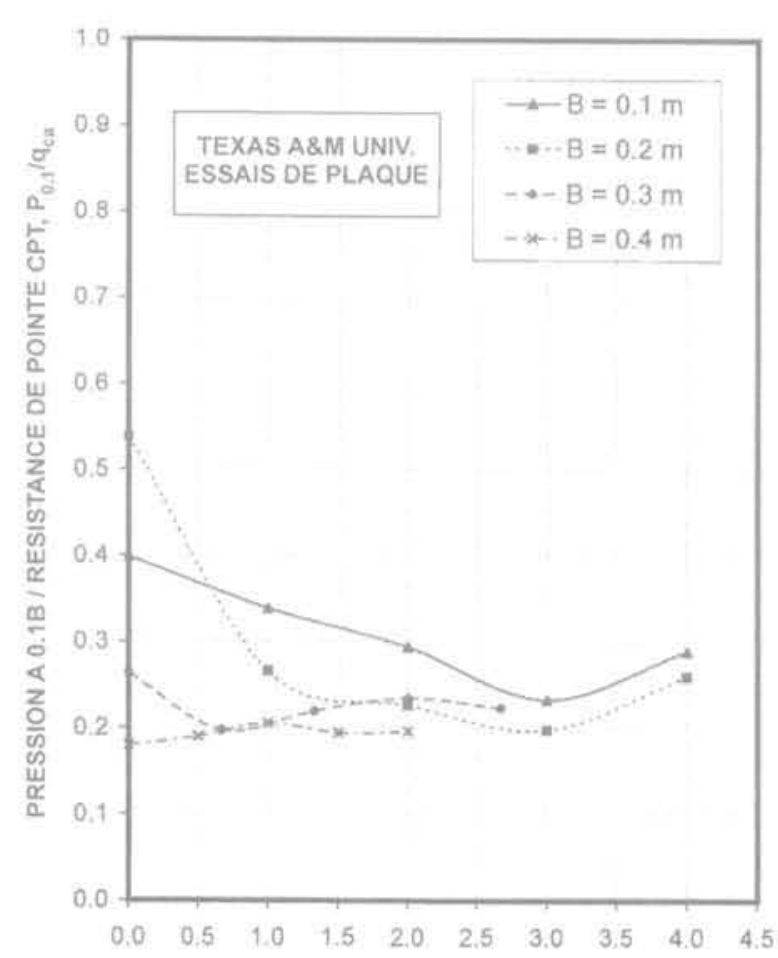

PROFONDEUR D'ENCASTREMENTILARGEUR DE LA PLAQUE, D/B

Fig. 8 Résultats des essais de plaque de Texas A\&M University (grandes déformations). Plate tests results for Texas A\&M University (large movements). 


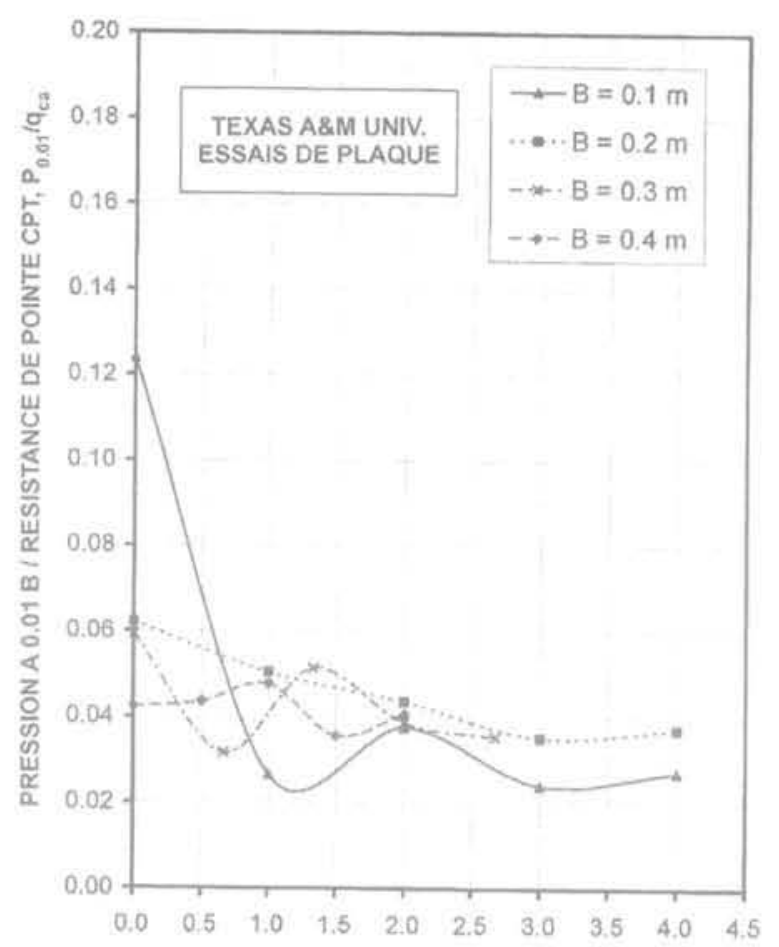

PROFONDEUR D'ENCASTREMENTILARGEUR DE LA PLAQUE, D/B

FiG.9 Résultats des essais de plaque de Texas A\&M University (petites déformations). Plate tests results for Texas A\&M University (small movements).
Les essais de fondations ont été conduits de la façon suivante. Les fondations ont été coulées en place et chargées selon la norme ASTM D1194-72. La charge verticale a été développée à partir de camions chargés et a été appliquée par paliers de $45 \mathrm{kN}$ jusqu'à la limite de $450 \mathrm{kN}$. Le tassement a été mesuré avec des extensomètres en prenant pour base une poutre.

L'effet de taille a d'abord été étudié en conduisant une série d'essais avec les fondations de $0,25 \mathrm{~m}, 0,5 \mathrm{~m}$, $0,75 \mathrm{~m}$ et $1 \mathrm{~m}$ encastrées à une profondeur de $1 \mathrm{~m}$. Les résultats présentés par Ismael (1985) sous la forme pression fonction du tassement sont représentés sur la figure 10 sous la forme pression fonction du rapport tassement/largeur, Les résultats montrent clairement que les courbes se regroupent en une courbe unique: donc, dans ce cas, l'effet de taille n'existe pas quand on présente les données de cette manière.

L'effet d'encastrement a été étudié ensuite en conduisant une série d'essais avec la fondation de $0,5 \mathrm{~m}$ encastrée à $0,5 \mathrm{~m}, 1 \mathrm{~m}, 1,5 \mathrm{~m}$, et $2 \mathrm{~m}$ de profondeur. Les résultats présentés par Ismael (1999) sous la forme pression fonction du tassement sont présentés sur la figure 11 sous la forme pression fonction du rapport tassement/largeur. Les résultats montrent clairement que les courbes se regroupent en une courbe unique; donc dans ce cas aussi, l'effet d'encastrement n'existe pas quand on présente les résultats de cette manière.

Dans ce sable où la résistance est constante avec la profondeur, l'effet de taille et d'encastrement sur les courbes p fonction du rapport s/B n'existe pas. Ces résultats confirment les résultats des essais de Texas A\&M University.
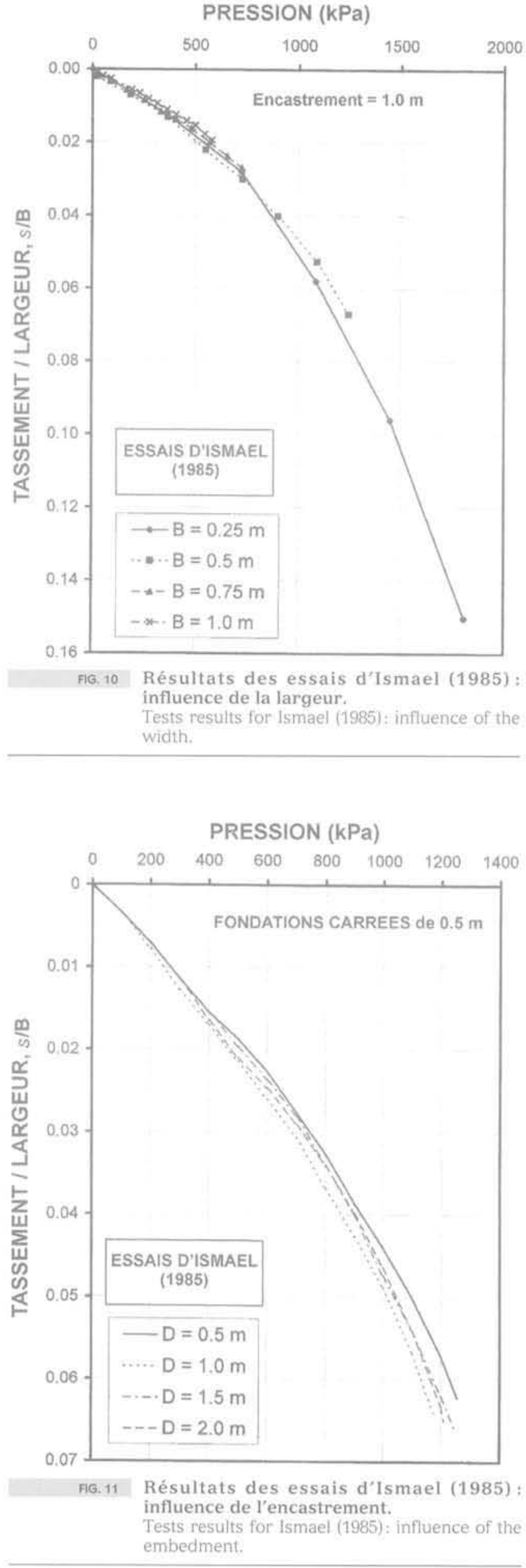


\section{7}

\section{Les essais de centrifugeuse Pu et Ko (1988)}

Pu et Ko (1988) ont conduit une série d'essais sur modèle réduit dans la centrifugeuse de l'Université du Colorado. Les modèles utilisés étaient une fondation carrée $(25,4 \mathrm{~mm} \times 25,4 \mathrm{~mm})$ et une semelle filante $(25,4 \mathrm{~mm} \times 152,4 \mathrm{~mm})$. Des essais ont été conduits à la surface du sable sec ainsi qu'en profondeur avec des rapports $\mathrm{D} / \mathrm{B}$ de $0,5,1,3$ et 5 . Le sable était uniforme et propre (SP) avec une densité relative élevée sur la profondeur de 0,3 m du conteneur de la centrifugeuse. Le sable a été mis en place par pluviation pour obtenir une densité uniforme. Le profil de résistance n'a pas été mesuré pendant les essais de 1988, mais des sondages CPT réalisés depuis sur du sable préparé de la même façon donnent une résistance de pointe qui augmente linéairement avec la profondeur (Ko, 1991, communication téléphonique) (Fig. 1a).

Les fondations ont été chargées avec un vérin hydraulique. La charge était donnée par une cellule de mesure de force et le tassement par un capteur LVDT. L'effet de taille a été étudié en utilisant la semelle filante et en conduisant des essais à $25 \mathrm{~g}, 50 \mathrm{~g}$ et $75 \mathrm{~g}$. Ces accélérations correspondent à des grandeurs prototypes de $0,63 \mathrm{~m}, 1,27 \mathrm{~m}$ et $1,90 \mathrm{~m}$. Les résultats présentés sous la forme de pression p fonction du rapport tassement-largeur s/B indiquent un effet de taille important (Fig. 12a). On peut aussi présenter les résultats sous la forme de pression divisée par la résistance moyenne du sol $\tau_{\mathrm{ff}}$ dans la zone influencèe par la fondation fonction du rapport s/B. La zone d'influence de la fondation est de l'ordre de deux fois la largeur pour les fondations carrées et quatre fois la largeur pour les semelles filantes. La valeur de $\tau_{\text {r }}$ est proportionnelle à l'accélération centrifuge, a, pendant l'essai car $\tau_{\mathrm{fa}}$ est proportionnel au niveau de contrainte; donc dans ce cas, au lieu de normaliser la pression par $\tau_{f,}$ on a simplement normalisé la pression par l'accélération centrifuge. La figure $12 \mathrm{~b}$ représente la courbe de $\mathrm{p} / \mathrm{a}$ en fonction de s/B; sur cette figure on voit que les courbes finissent par se regrouper dans une bande beaucoup plus serrée. Ceci montre que, lorsque la résistance du sol augmente avec la profondeur (Fig. 1a), l'effet de taille est réduit de façon importante, une fois que la pression sous la fondation est normalisée en la divisant par la résistance du sol dans la zone d'influence.

L'effet d'encastrement a été étudié en utilisant la fondation carrée et en conduisant des essais à des profondeurs relatives $\mathrm{D} / \mathrm{B}$ égales à $0,0,5,1,3$ et 5 . Les essais ont tous été réalisés à $50 \mathrm{~g}$ ce qui correspond à une fondation prototype de $1,27 \mathrm{~m}$ de côté. Pu et Ko (1988) présentent les résultats sous la forme de p en fonction de s/B (Fig. 13a); sous cette forme, l'influence de l'encastrement est claire. On peut aussi présenter les résultats sous la forme $\mathrm{p} / \tau_{\mathrm{f}}$ en fonction de $\mathrm{s} / \mathrm{B}$. Dans ce cas, on choisit $\tau_{\text {fa }}$ comme etant la résistance moyenne dans la zone ou profondeur d'influence de la fondation; cette profondeur est égale à deux fois la largeur B de cette fondation carrée. On a donc pris la résistance moyenne comme étant celle qui existe à une profondeur de $1 \mathrm{~B}$ sous la fondation $(\mathrm{z}=1 \mathrm{~B}$ pour $\mathrm{D} / \mathrm{B}=0$ et $\mathrm{z}=$ $6 \mathrm{~B}$ pour $\mathrm{D} / \mathrm{B}=5$ ). La figure $13 \mathrm{~b}$ représente la variation

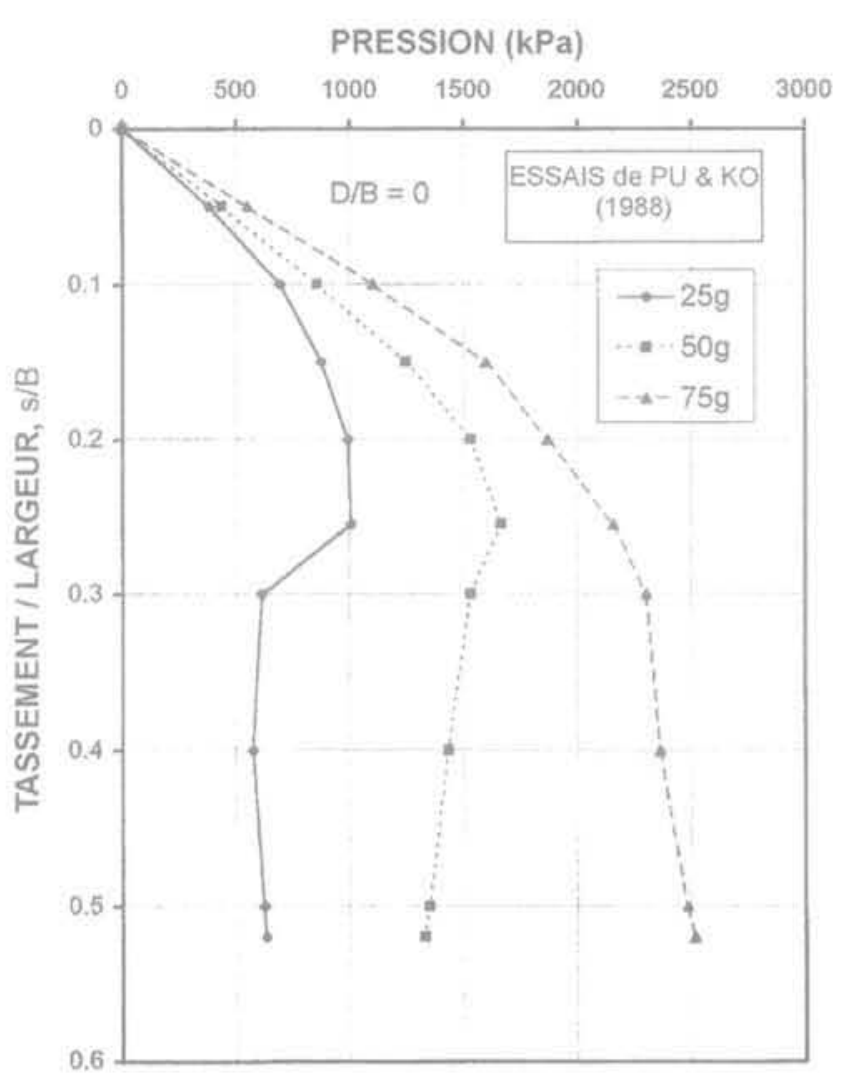

(a)

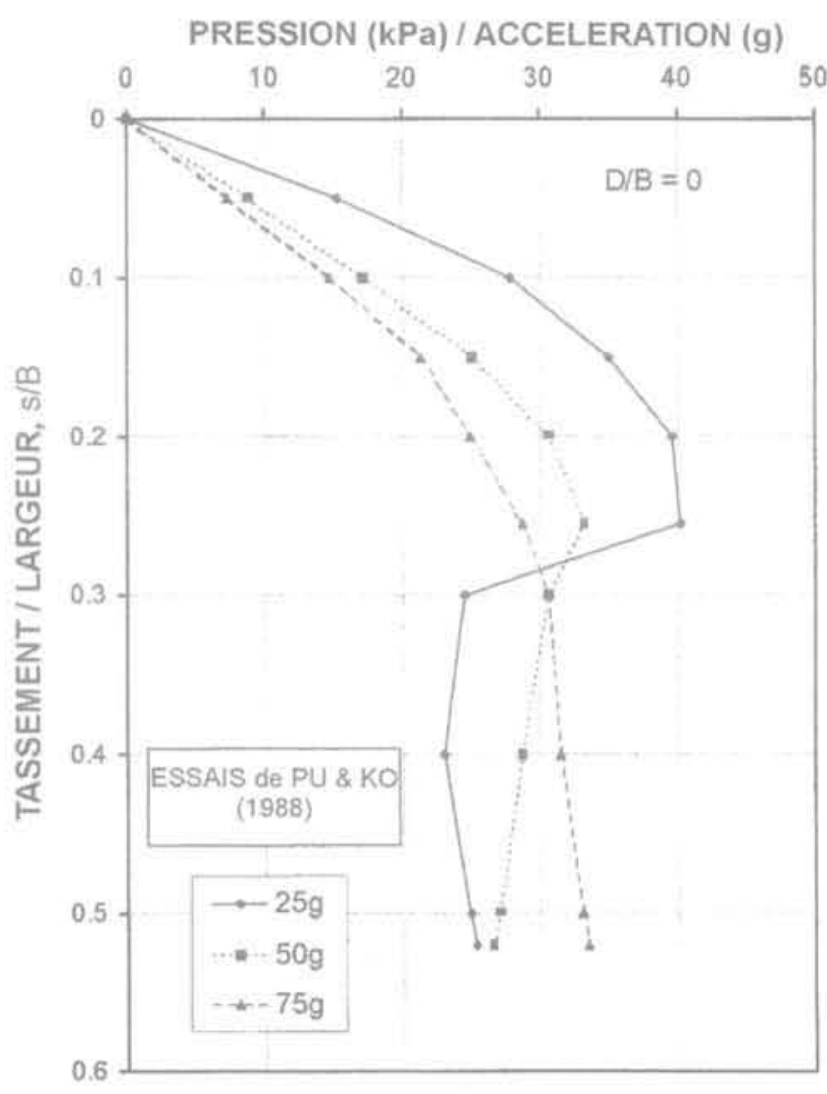

(b)

FG. 12 Résultats des essais en surface de Pu et Ko (1988) : (a) avant normalisation, (b) après normalisation. Results of surface tests for Pu and Ko (1988): (a) before normalization, (b) after normalization. 
de $p /((D / B)+1)$ en fonction de $s / B$; dans ce cas, l'effet d'encastrement disparait pratiquement. Ceci indique que lorsque la résistance du sol augmente avec la profondeur (Fig. 1a), l'effet d'encastrement disparait pratiquement dès que la pression sous la fondation a été normalisée avec la résistance moyenne du sol dans la zone d'influence.

\section{8}

\section{Les essais de la Federal Highway Administration (Lutenegger, 1995)}

La Federal Highway Administration (FHWA) a réalisé un grand nombre d'essais de fondation dans une fosse de $7,1 \mathrm{~m} \times 6,5 \mathrm{~m} \times 3,5 \mathrm{~m}$ de profondeur remplie de sable au Turner-Fairbank Highway Research Center près de Washington DC sous la direction de Al DiMillio et de Mike Adams. Une des séries d'essais a été réalisée pour étudier l'effet d'encastrement $\mathrm{D} / \mathrm{B}(0,0,5$ et 1) avec une fondation carrée de $0,91 \mathrm{~m}$ de côté (Lutenegger, 1995, communication personnelle). Le sable est un sable propre uniforme et humide placé par couche de 0,3 m d'épaisseur et compacté jusqu'à une densité relative moyenne. Une série d'essais pressiométriques (PMT) a été réalisée par Barfknecht et Briaud (1999) dans la fosse d'essai et a donné un profil de pression limite qui augmente avec la profondeur (Fig. 14). Pendant les essais PMT, la nappe était à une profondeur de $2,1 \mathrm{~m}$; il n'y avait pas de nappe pendant les essais de chargement des fondations.

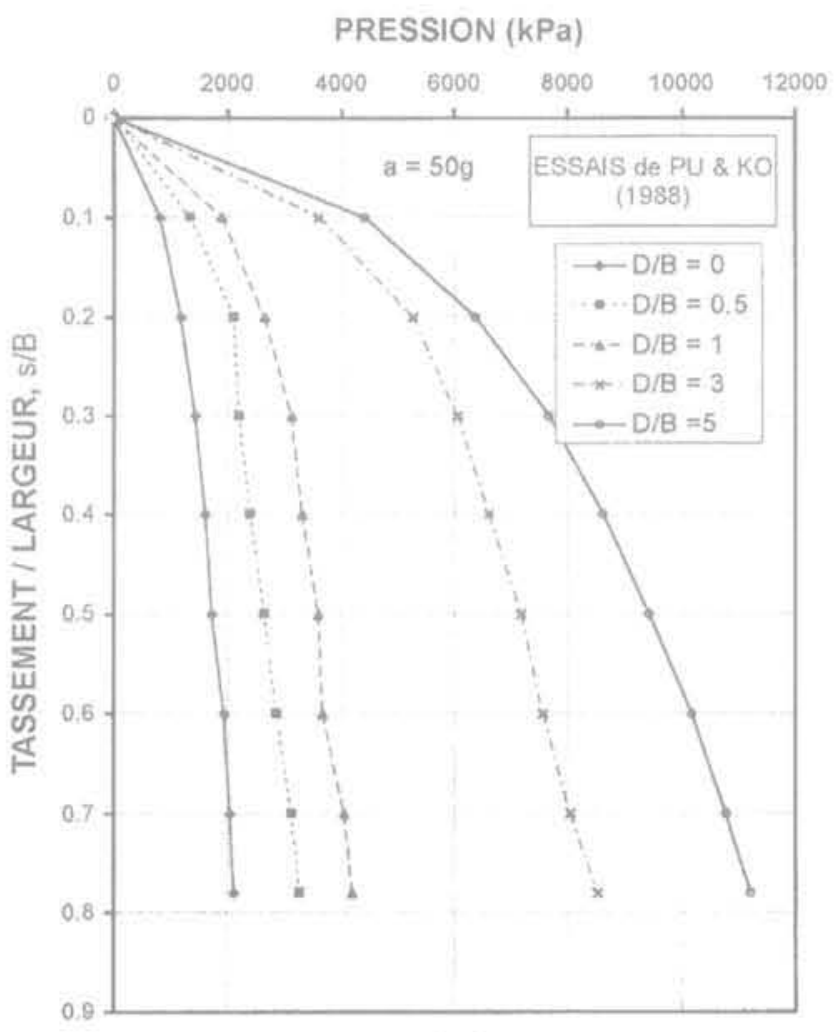

(a)
Les fondations en béton ont été charqées par paliers, la charge a été mesurée par une cellule de mesure de force et le tassement comme la moyenne des lectures de quatre LVDTs placés à chaque coin de la fondation. Les courbes de pression p fonction du rapport tassement/largeur s/B pour les trois profondeurs d'encastrement sont présentées sur la figure $15 \mathrm{a}$. Cette figure indique un effet d'encastrement important qui disparait pratiquement quand les courbes sont tracées sous la forme $p / p_{1}$ en fonction de $s / B$ (Fig. 15b): $\mathrm{p}_{\mathrm{u}}$ est la pression limite moyenne du PMT dans la zone de profondeur égale à $1 \mathrm{~B}$ sous la fondation.

Ces essais indiquent que, lorsque la résistance du sol augmente avec la profondeur, l'effet d'encastrement disparait pratiquement dès qu'on normalise la courbe par rapport à la résistance moyenne du sol dans la zone influencée: $\mathrm{p} / \mathrm{p}$. en fonction de $s / \mathrm{B}$.

\section{9}

\section{Les essais des Laboratoires des ponts et chaussées (Khebib, Canépa, Magnan, 1997)}

Les Laboratoires des ponts et chaussées (LPC) ont réalisé, entre 1978 et 1990, une grande série d'essais de fondations superficielles sur site (Canépa, Despreles, 1990). Grâce à une entente de coopération entre la FHWA (Al DiMillio) et les LPC (Jean-Pierre Magnan), les LPC ont incorporé les données des 51 essais de fondations réalisés par les L.PC dans la base de données FHWA pour fondations superficielles et a produit un

PRESSION / (D/B+1) (kPa)

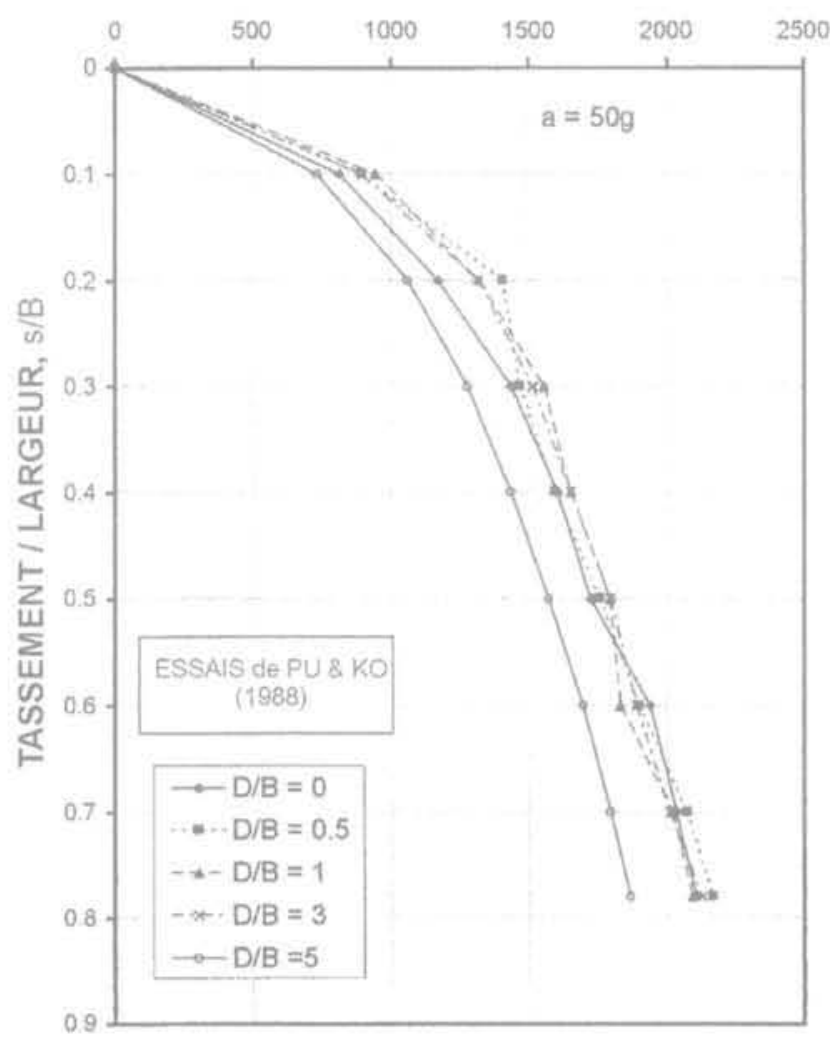

(b)

FiG. 13 Résultats des essais en profondeur de Pu et Ko (1988) : (a) avant normalisation, (b) après normalisation. Results of the tests at depth for Pu and Ko (1988): (a) before normalization, (b) after normalization. 


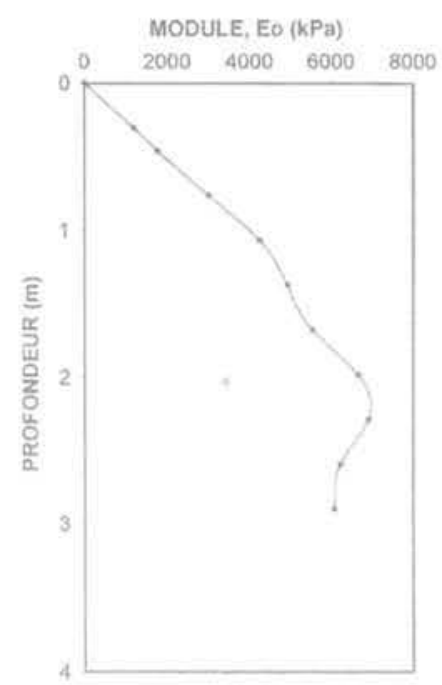

(a)
PRESSION LIMITE, $P$, (KPa)

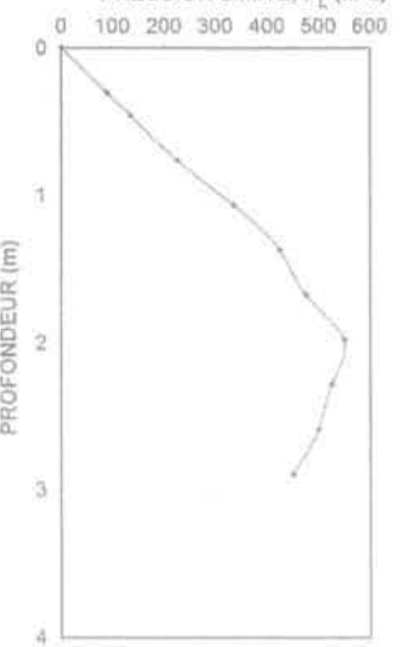

(b)
FIG, 14 Résultats des essais pressiométriques dans la fosse de FHWA.

Pressuremeter tests results in the FHWA sand pit.

document (Khebib, Canépa, Magnan, 1997). C'est ce document qui constitue la base de ce qui suit.

Le sable du site de Labenne est un sable propre fin et moyennement dense (SP) dans la zone d'influence des essais. Le nombre moyen de coups de battage au SPT dans cette zone est de 15 coups $/ 0,3 \mathrm{~m}$. La résistance du

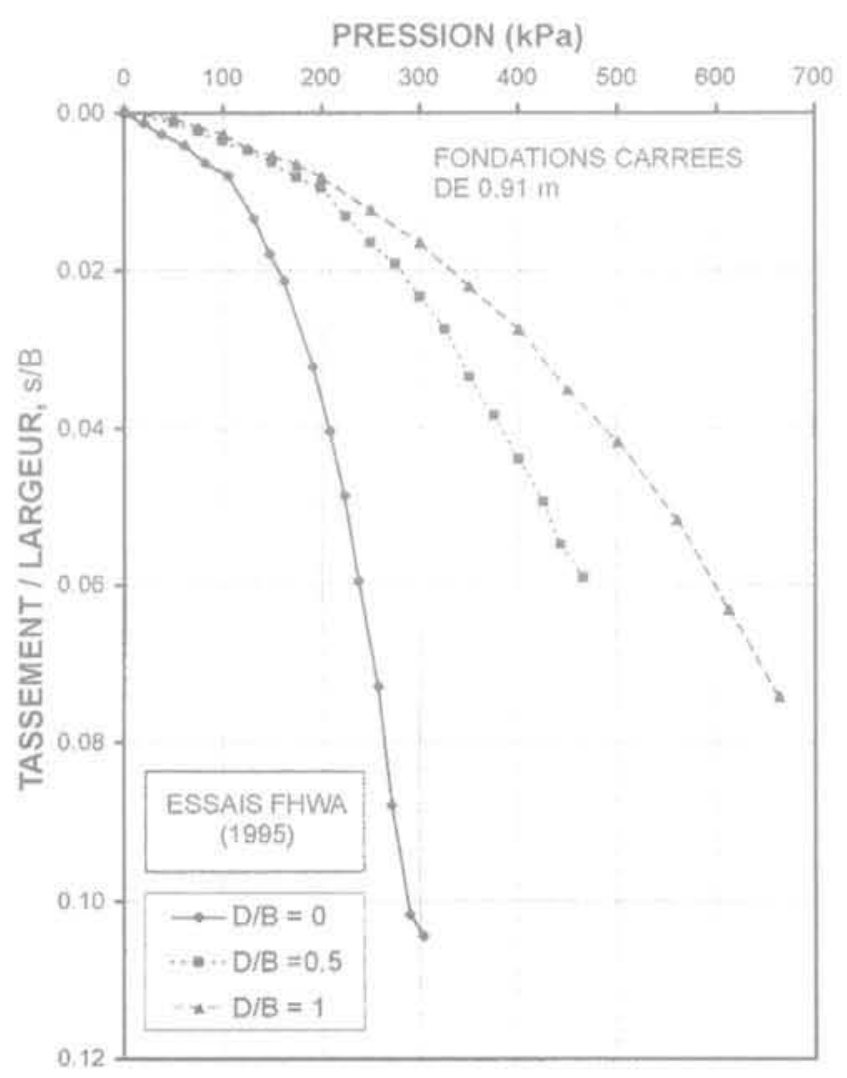

(a) sol augmente légèrement de 0 à $3 \mathrm{~m}$ et est caractérisée par l'équation (Khebib, Canépa, Magnan, 1997):

$$
\mathrm{p}_{\mathrm{L}}(\mathrm{kPa})=780(1+0,15 \mathrm{z}(\mathrm{m}))
$$

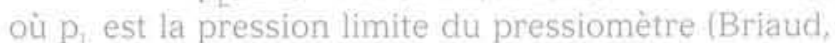
1992) et z est la profondeur.

Les fondations étaient carrées et mesuraient $0,7 \mathrm{~m}$ $\times 0,7 \mathrm{~m}$ et $1 \mathrm{~m} \times 1 \mathrm{~m}$. Les essais de chargement ont été réalisés par paliers égaux au 1/10 de la charge ultime prévue et maintenue pendant une heure. On peut évaluer l'effet de taille en comparant les résultats des essais faits près de la surface $(D=0,2 \mathrm{~m}$ ) pour la fondation de $0,7 \mathrm{~m}$ et de $1 \mathrm{~m}$. Les courbes donnant la pression $\mathrm{p}$ en fonction du rapport tassement/largeur (s/B) de la fondation sont regroupées sur la fiqure 16a. D'après cette figure l'effet de taille est tres faible, On peut aussi tracer les courbes sous la forme de $p / p_{1}$ en fonction de $s / B$ (Fig. 16b) où p est la moyenne de la pression limite au pressiomètre dans la zone d'influence de la fondation. II y a peu de différence entre la figure $16 \mathrm{a}$ et $16 \mathrm{~b}$ parce que la résistance du sol est pratiquement constante avec la profondeur.

L'effet d'encastrement peut être évalué en comparant les résultats de la fondation de $1 \mathrm{~m}$ chargée à deux profondeurs différentes: $0,2 \mathrm{~m}$ et $1 \mathrm{~m}$. Les courbes $\mathrm{p}$ fonction de $\mathrm{s} / \mathrm{B}$ pour ces essais sont présentées sur la figure 17 a. Cette figure indique une petite influence de l'encastrement qui décroit quand on trace les courbes en $\mathrm{p} / \mathrm{p}$. fonction de $\mathrm{s} / \mathrm{B}$ (Fig. 17b): $\mathrm{p}_{1}$ est la pression limite moyenne au pressiometre dans la zone de profondeur égale à $1 \mathrm{~B}$ sous la fondation.

Ces essais indiquent que lorsque la résistance du sol est pratiquement constante avec la profondeur, l'effet de taille et l'effet d'encastrement sont négligeables.

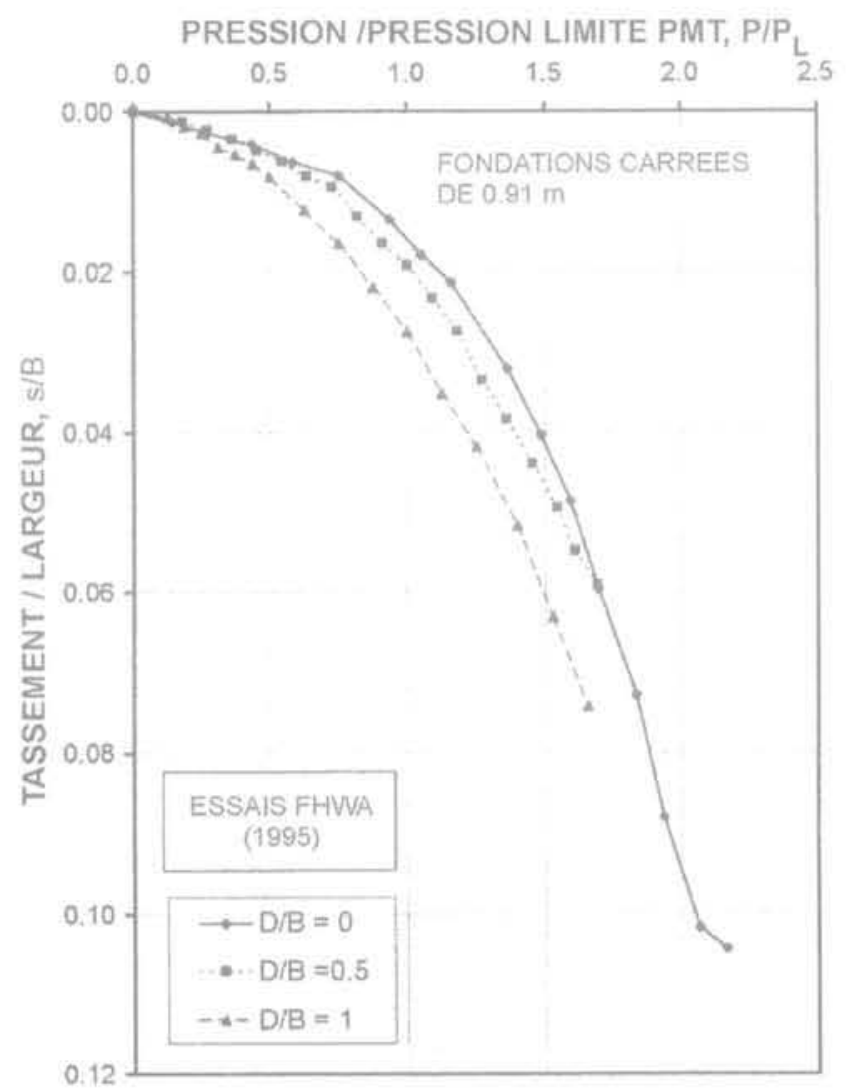

(b)

FIG, 15 Résultats des essais FHWA (Lutenegger, 1995) : (a) avant normalisation, (b) après normalisation. Results of the FHWA tests (Lutenegger, 19950) : (a) before normalization, (b) after normalization. 


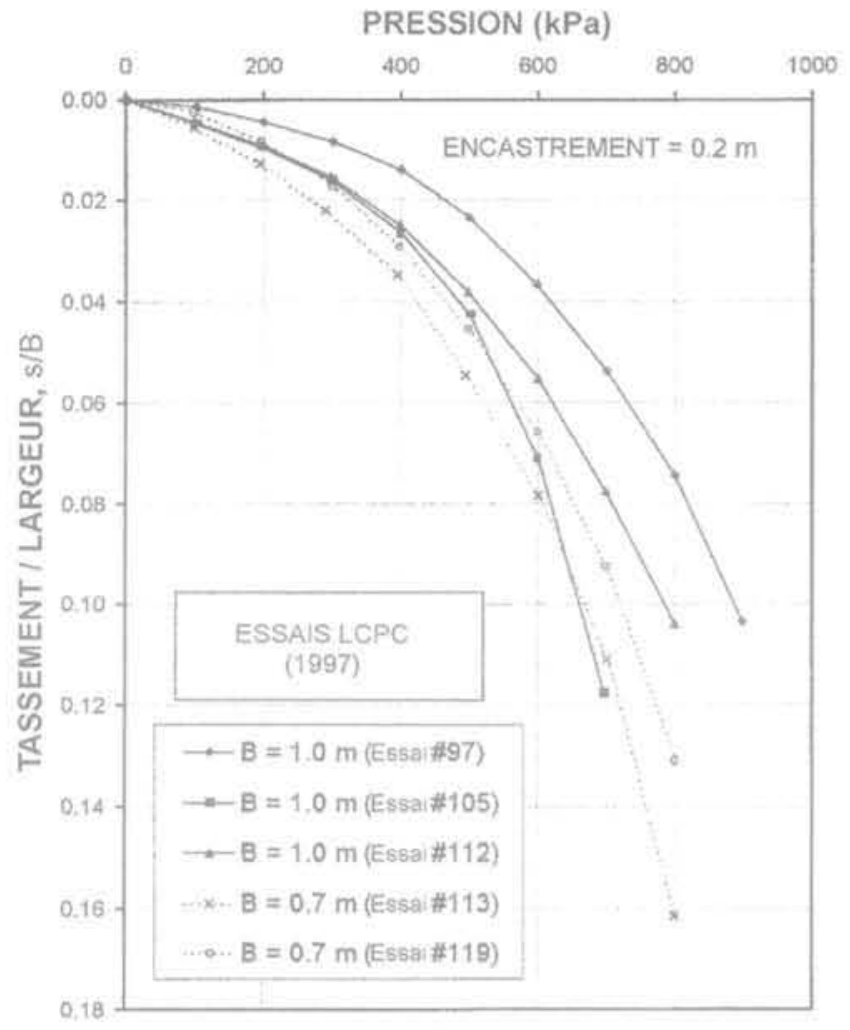

(a)

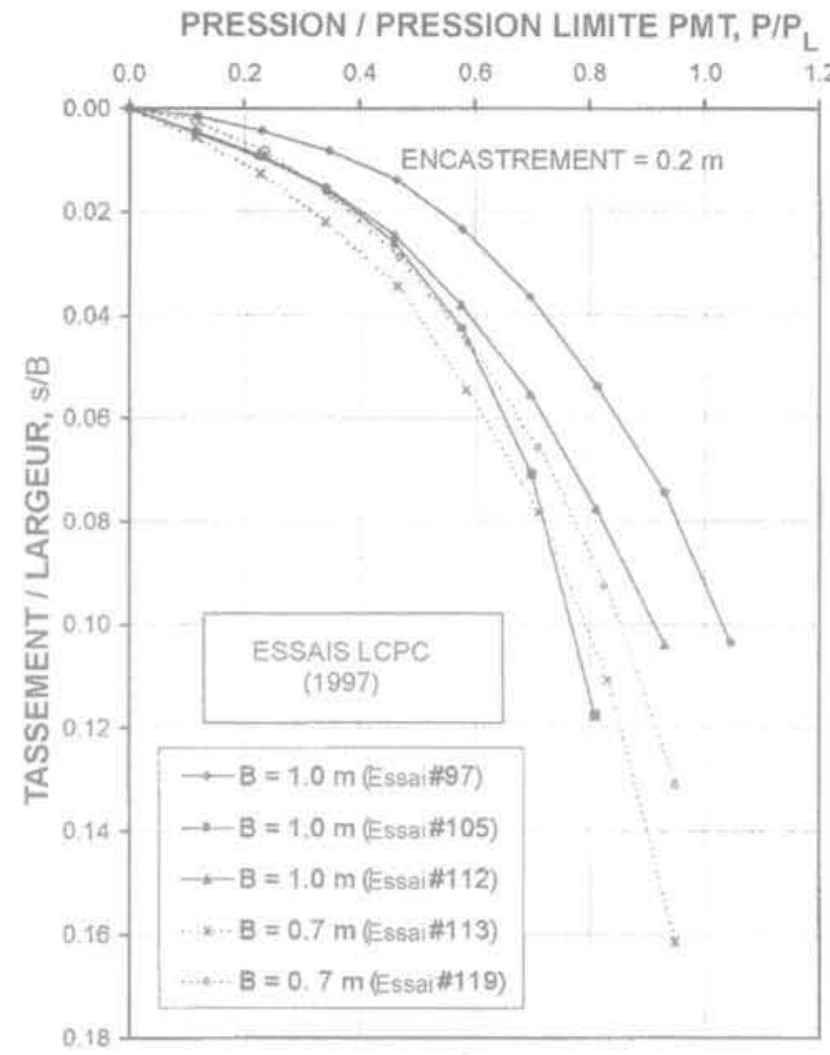

(b)

59. 16 Résultats des essais LCPC près de la surface (Khebib, Canepa, Magnan, 1997) : (a) avant normalisation, (b) après normalisation.

Results of the LCPC tests near the surface (Khebib, Canepa, Magnan, 1997): (a) before normalization, (b) after normalization.

\section{Les essais de Consoli et al. (1998)}

L'université fẻdérale de Rio Grande Do Sul au Brésil a réalisẻ des essais de plaques et de fondations sur un sol résiduel. Le sol est une argile sableuse et silteuse jusqu'à $3,5 \mathrm{~m}$ de profondeur. Le profil de résistance a été caractérisé avec le pénétromètre statique et indique une résistance approximativement constante avec la profondeur dans la zone d'influence des fondations. La valeur moyenne de la résistance de pointe est égale à $500 \mathrm{kPa}$. Des essais en laboratoire ont donné la résistance en compression simple du sol.

Les fondations étaient des plaques circulaires de $0,3 \mathrm{~m}$ à $0,6 \mathrm{~m}$ de diamètre et des semelles carrées de $0,4 \mathrm{~m}$ à $1 \mathrm{~m}$ de cote. Les essais de chargement ont été réalisés en suivant la norme brésilienne MB-3472 (Foundations, 1991) et par palier d'au moins 30 minutes. Tous les essais ont été faits au fond de fausses de 1,2 m de profondeur. On peut évaluer l'effet de taille en comparant les résultats de tous les essais. La figure 18a montre les courbes pression-tassement obtenues par Consoli et ses collègues. La figure $18 \mathrm{~b}$ montre les résultats aussi présentés par Consoli après normalisation de la pression avec la résistance à la compression simple du sol sous la fondation. Les courbes de la figure 18a sont dispersées tandis que les courbes de la figure $18 \mathrm{~b}$ sont regroupées dans une plage étroite.
Ces essais indiquent que lorsque la résistance du sol est pratiquement constante avec la profondeur, l'effet de taille est négligeable.

\section{1}

\section{Discussion}

Les équations discutées et les données expérimentales présentées ont montré que le profil de résistance du sol en fonction de la profondeur a, pour les fondations dans le sable, un impact direct sur l'effet de taille et l'effet d'encastrement. Les données expérimentales montrent que si la résistance du sol est constante avec la profondeur (Fig. 1b), l'effet de taille et l'effet d'encastrement sur la courbe pression fonction du rapport tassement/largeur sont négligeables (essais TAMU, Ismael, LPC, Consoli). Si la résistance du sol augmente proportionnellement à la profondeur (Fig. 1a), alors il y a un effet de taille et un effet d'encastrement sur la courbe pression fonction du rapport tassement/largeur; cependant ces deux effets deviennent négligeables pour la courbe du rapport pression-résistance du sol dans la zone influencée par la fondation fonction du rapport tassement/largeur (essais Pu et Ko, FHWA). Ces observations représentent une indication que la courbe du rapport pression/résistance $\left(\mathrm{p} / \tau_{\mathrm{f}}\right)$ fonction du rapport tassement/largeur $(\mathrm{s} / \mathrm{B})$ est unique pour un sol donné et pour des fondations carrées chargées ver- 


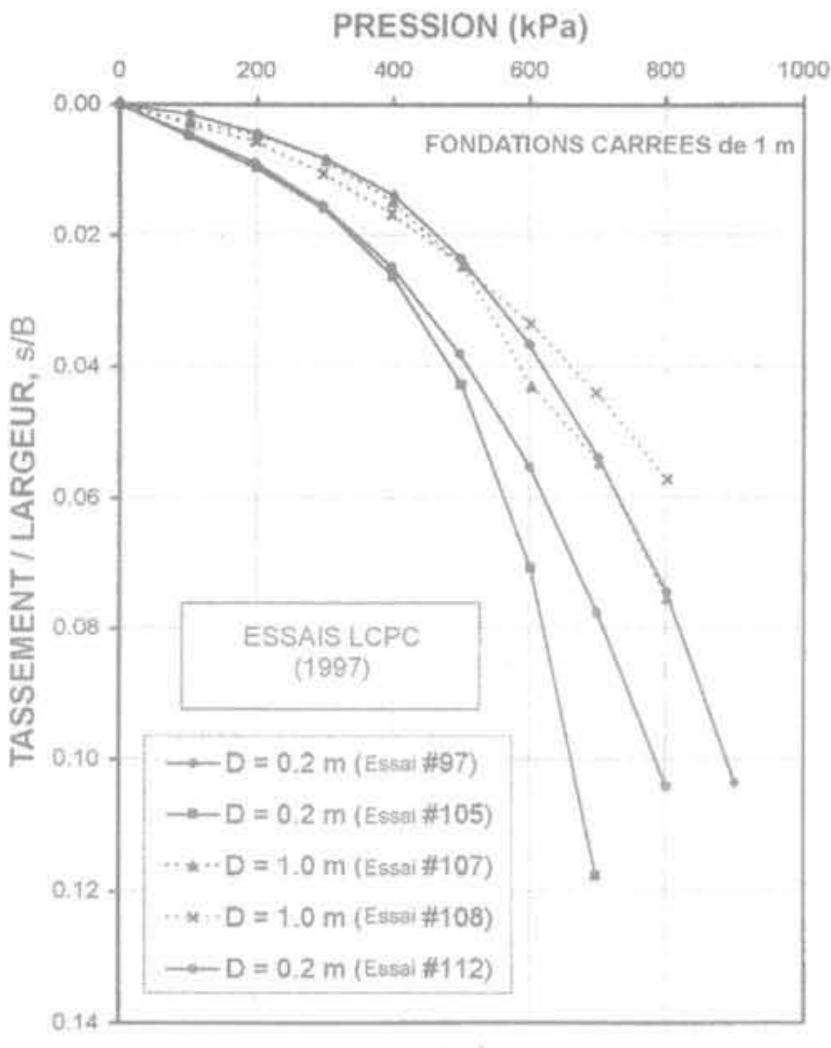

(a)

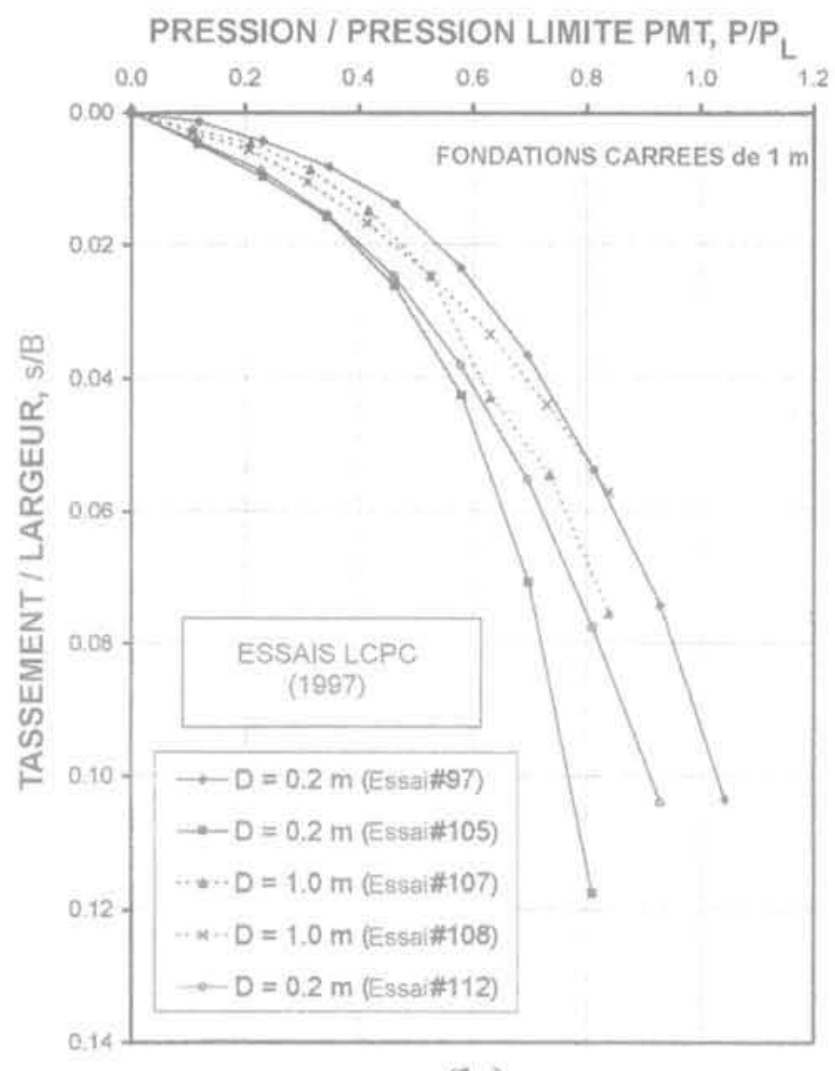

(b)

FG. 17 Résultats des essais LCPC en profondeur (Khebib, Canepa, Magnan, 1997): (a) avant normalisation, (b) après normalisation.

Results of the LCPC tests at depth (Khebib, Canepa, Magnan, 1997): (a) before normalization, (b) after normalization.

ticalement en leur centre sur une surface horizontale. L'unicité de cette courbe est la base de la méthode proposée par Briaud et Jeanjean (1994). Cette méthode est basée sur l'unicité de la relation entre le rapport pression sous la fondation divisée par la pression correspondante obtenue au pressiomètre, d'une part, et le rapport tassement divisé par la largeur de la fondation, d'autre part.

Si la courbe $\mathrm{p} / \tau_{\mathrm{ta}}$ fonction de $\mathrm{s} / \mathrm{B}$ est unique, est-ce que l'équation générale de capacité portante ultime décrit le phénomène correctement? La réponse est out dans le cas où la résistance du sol augmente proportionnellement à la profondeur car, dans ce cas, les hypothèses sont bien vérifiées. En effet, un angle de frottement constant et une contrainte effective qui augmente proportionnellement à la profondeur correspondent bien à ce cas. Par contre la réponse est non. pour tous les autres cas, y compris le cas d'une résistance de sol constante en fonction de la profondeur. Dans ce dernier cas, la capacité portante ultime est indépendante de la grandeur de la fondation et de l'encastrement. Ceci est contraire à l'équation de capacité portante ultime et rend le coefficient $\mathrm{N}$, fonction de la largeur B de la fondation (Vesic, 1973) et $\mathrm{N}_{\mathrm{q}}$ fonction de la profondeur d'encastrement.

On doit noter que le profil de résistance constante avec la profondeur correspond au cas des quatre sites naturels (TAMU, Ismael, L.PC, Consoli) tandis que le profil de résistance augmentant linéairement avec la profondeur correspond au cas des deux dépôts de sable artificiels (Pu et KO. FHWA). Ceci indique que les profils constants sont en réalité peut-être plus com- muns et les expériences en laboratoire devraient refléter ce type de profil.

\section{2}

\section{Conclusion}

Le cas considéré est celui de fondations carrées dans du sable et soumises à des charges verticales appliquées au centre de la fondation reposant sur un terrain plat horizontal. La pression moyenne sous la fondation est $\mathrm{p}$, le tassement est $\mathrm{s}$, la largeur de la fondation est B, la profondeur d'encastrement est D et la résistance moyenne du sol dans la zone d'influence de la fondation est $\tau_{\mathrm{fa}}$. La question posée est la suivante: y a-t-il un effet de taille et un effet d'encastrement dans ce cas? La réponse et les conclusions qui suivent sont bien évidemment limitées par les données expérimentales utilisées dans cet article, par les types de sols représentés sur ces sites expérimentaux, et par le raisonnement présenté. Les données expérimentales ont été obtenues pour des fondations d'une taille variant de $0,25 \mathrm{~m}$ à $3,0 \mathrm{~m}$ de côté et pour des encastrements relatifs $\mathrm{D} / \mathrm{B}$ variant de 0 à 5 . Le raisonnement fait appel à l'équation classique de capacité portante uitime et à la théorie de l'élasticité:

1) il n'y a pas d'effet de taille ni d'effet d'encastrement pour la courbe $\mathrm{p} / \tau_{\mathrm{fa}}$ fonction de $\mathrm{s} / \mathrm{B}$ quel que soit le profil de résistance du sol;

2) il n'y a pas d'effet de taille ni d'effet d'encastrement pour la courbe p fonction de s/B quand le profil de résistance est constant avec la profondeur; 

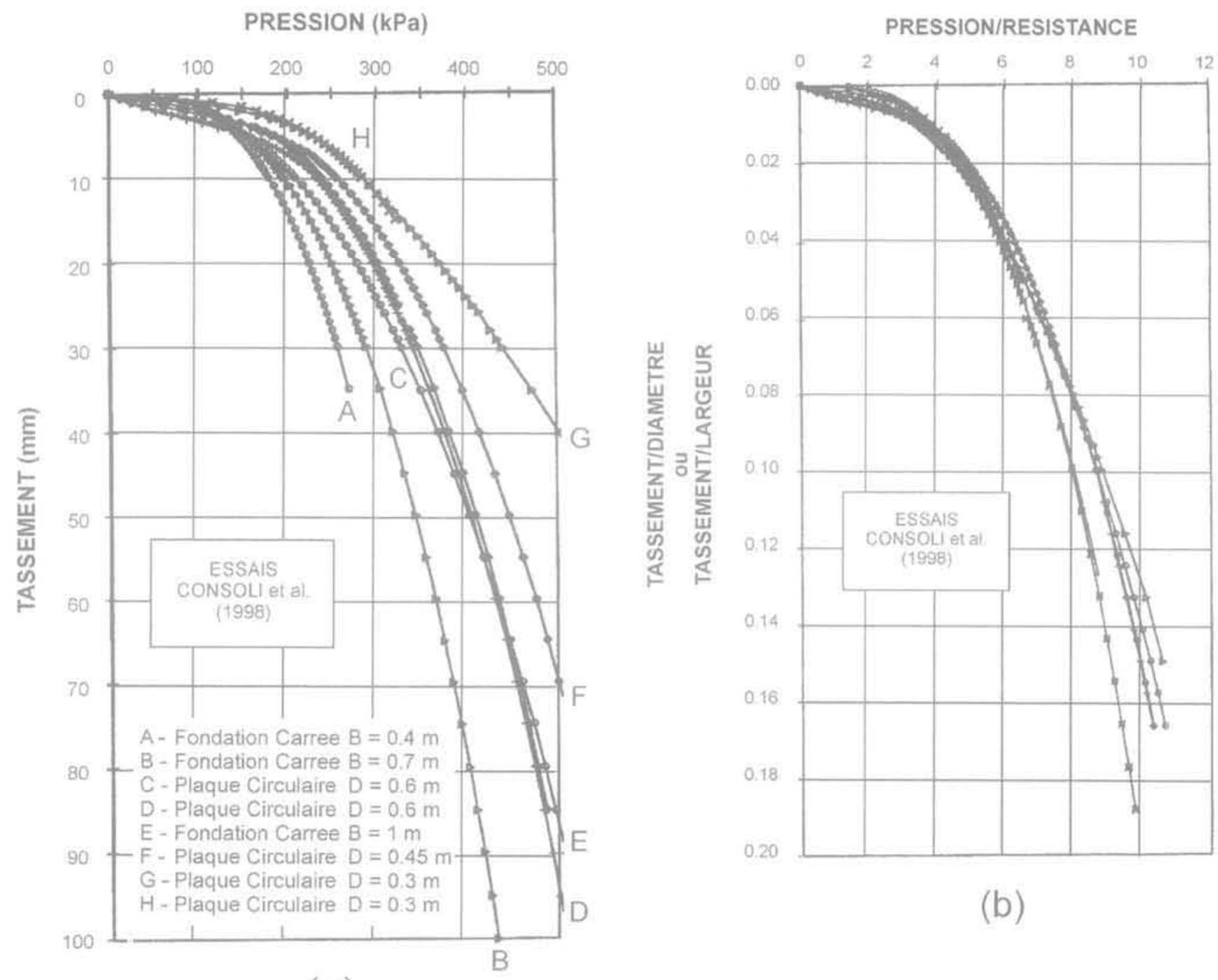

(b)

(a)

FIG. 18 Résultats des essais de Consoli (Consoli et al., 1998) : (a) avant normalisation, (b) après normalisation. Results of the tests by Consoli (Consoli et al., 1998): (a) before normalization, (b) after normalization.

3) il y a un effet de taille et un effet d'encastrement pour la courbe $\mathrm{p}$ fonction de s/B quand le profil de résistance du sol n'est pas constant avec la profondeur. L'effet correspond à une augmentation ou à une diminution selon que la résistance du sol augmente ou diminue avec la profondeur;

4) l'équation générale de capacité portante ultime dans les sables correspond à un profil de résistance de sol qui augmente linéairement avec la profondeur car $\phi^{\prime}$ et $\gamma$ sont constants et le sol est sec. Dans ces conditions,

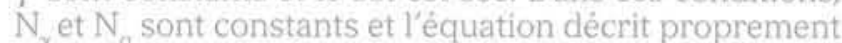
l'influence de B et de D. Pour tout autre profil de résistance, cette équation ne représente pas la vraie variation de la capacité portante ultime car les hypothèses, sur lesquelles elle est basée, ne sont pas vérifiées.

\section{REMERCIEMENTS}

Nous tenons à remencier la Federal Hiohway Administration (FHWA) qui a subventionné ce projet et Al DiMillio et Mike Adams de la FHWA pour leur coopération. Nous tenons aussi à remercier Jean-Pierre Magnan et Yves Canépa (Laboratoires des Ponts et chaussees), Nabil Ismae! (Kuweit University), Hon-Yim Ko (Université du Colorado, Boulder) et Alan Lutenegger (Université du Massachusetts) pour leurs contributions à ces travaux de recherche. Les essais de plaques à Texas A\&M University ont été réalisés par Fugro Geosciences (Recep Yilmaz). De nombreux anciens élèves ont contribué à cette recherche. On mentionne tout particulièrement Jayson Barfknecht (essais de plaque, TAMU), Jonqhyub Lee (recherche bibliographique), Bob Gibbens et Philippe Jeanjean (essais sur grandes fondations, TAMU) et Remon Melek (recherche bibliographique). Nous remercions aussi Louis Briaud pour sa révision du manuscrit. 
Barfknecht J. Briaud J.L. - « Effect of Scale and Depth of Embedment for Footings in Sand x. Research Report, Department of Civil Engineering. Texas A\&M University; College Station, Texas, USA. 1999.

Bolton M.D., Lau C.K. - $\alpha$ Scale effects in the bearing capacity of granular soils is. 12th Jint. Conf. of Soil Mechanics and Foundation Engineering, vol. 2, Rio. 1989, p. 895-898.

Bolton M.D. - aThe Strength and Dilatancy of Sands n. Géotechnique, Londion, vol. $36, n^{\circ} 1,1986$, p. 65-78.

Brazilian Standards MB-3472 - Foundations - Static loading tests, 1991.

Briaud J.L: - The Pressuremeter. A.A. Bal. kema Publishers, Brookfield, Vermont. USA. 1992, 322 p.

Briaud J.L. Gibbens R.M. - Large Scale Load Tests and Data Base of Spread Footings on Sand. Federal Highway Admihistration, Publication No. FHWA-RD97-068, McLean, Virginia, USA 1997. $220 \mathrm{p}$.

Briaud J.L... Gibbens, R.M. - « Behavior of five Large spread Footings in Sand $n$. Journal of Geotechnical and Geoenvinunmental Engineering, vol. 125, $n^{\circ}$ 9, American Society of Civil Engineers, Reston. Virginia, USA, Sept, 1999.

Briaud I.I. Gibbens R.M. (Eds) - re Predicted and Measured Behavior of Five Spread Footings on Sand x, Geotechnical Special Publication $n^{\circ} 41$. American Society of Civil Engineers, Reston, Virginia, USA, 1994, 256 p.

Briaud J.1., Jeanjean P. - $\alpha$ Load Settlement Curve Method for Spread Footings on Sand v. Geotechrical Special Publication $\mathrm{n}^{\circ} 40$, Ed. A.T. Yeung and G.Y. Felio. American Society of Civil Engineers. Reston, Virginia, USA, 1994, p. 1774 1804.

Canépa Y., Despresles D. - Catalogue des essais de chargement de fondations superficielles réalisés sur sites par les LPC (1978-1990). FAER1.17.020, LREP Melun, décembre 1990.

Consoli N.C. Schnaid F. Milititsky J."Interpretation of Plate Load Tests on Residual Soil Site s. Journal of Geotechnical and Geoenvironmental Engineering, vol. 124, $n^{\circ}$ 9, Sept. 1998, p. 857 867 .

Corté J.F. - i Essais sur modèles réciuits en géotechnique in, Proceedings 12th lnt. Cont. of Soil Mechanics and Foundation Engineering, vol, 4, Rio, 1980, p. 2553. 2571

De Beer E.E. - $a$ The Scale Effect on Phe nomenon of Progressive Bupture in Cohesionless Soils v, Proc, 6th Int. Conf. Soil Mechanics and Foundation Engineering. Univ. of Toronto, Canada, vol. 2 , 1965, p. 13-17.

Garnier 1. - " Validation des modèles physiques et numériques : problème des effets de taille n. ICSMFE, Hambourg, vol. 3, 1997, p. 659-663.

Graham J. Hovan. J.M - u Stress Characteristics for Bearing Capacity in Sand Using a Critical State Model n. Can. Geotech. J., Ottawa, vol. 23, 1966, p. 195-202.

Habib P. - \& Effet de taille et surfaces de glissement w. Revue française de géotechnique, 31, 2v trimestre 1985, p. 5-10.

Hettler A., Gudehus G. - a Influence of the Foundation Width on the Bearing Capacity Factorn. Soils and Foundations, vol. 28, n 4. Dec. 1988, p. 81-92.

Ismael N.F. - ". Allowable Pressure from Loa ding Tests on Kuwaiti Solis x. Can. Geotech. J., n 22, Ottawa, 1985; p. 151-157.

Khebib Y.. Canépa Y., Magnan J.P. - Base de Données de Fondations Superficielles SHALDB ; essais des Laboratoires des ponts et chaussées. Laboratoire Centra des Ponts et Chausseees, Paris, 1997.

Kimura T. Kusakabe O, Saitoh K. - a Geotechnical Moclel Tests of Bearing Capacity Problems in a Centrifuge o. Géo- technique, London, vol. $35, n^{2} 1$, 1985, p. 33-45.

Lutenegger A. - Communication personnelle du 27-9-1995 (by fax dated 9-27-95).

Mayne P.W., Poulos, H.G. - "Approximate Displacement Influence Factors for Elastic Shallow Foundations n. Journal of Geotechnical and Geoenvironmental Engineering, vol. 125, n 6. 1999, p. 453460.

Ovesen N.K. - "Centrifugal Testing Applied to Bearing Capacity Problems of Footings on Sand in. Geotechnique, London, vol. $25, n^{\circ} 2,1975, p .354-401$.

Perkins S.W.. Madson C.R. - " Bearing Capacity of Shallow Foundations on Sand: A Relative Density Approach o. Journal of Geotechnical and Geoenvironmental Engineering, vol. $126, n^{\circ} 6,2000$, p. $521-530$

Pu J.L. Ko, H.Y. - "Experimental Determi. nation of Bearing Capacity in Sand by Centrifuge Footing Tests o, Proceed. of Centrifuge '88, J.F. Corté Ed.. A.A. Balkema Publishers; Rotterdam. Netherlands, 1988, p. 293-299.

Shiraisi S. - "Variation in bearing capacity factors of dense sand assessed by model loading tests p. Soils and foundations, 1 . val, 30, march 1990, p. 17-26.

Steenfelt J.S. - "Scale effect on bearing capacity factor Ny". Proceed. 9th Int. Conf. Soil Mechan. and Found. Eng.. Tokvo, vol. 1. 1977 . p. 749-752

Terzaghi K., Peck R.B., Mesri G. - "Soil Mechanics in Engineering Practice w, John Wiley and Sons, New York, 1996, 3rd ed. p. 549.

Vesic A.S. - "Analysis of Ultimate Loads of Shallow Foundations v. Journal of the Soil Mechanics and Foundations Division, vol. 99, SM1, Jan. 1973, p. 45-73.

Yamaquchi H. Kimura T. Fuii N. - v On the Influence of Progressive Failure on the Bearing Capacity of Shallow Foundations in Dense Sand D. Soils and Foundations, vol. 16, $n^{\circ}$ 4, Dec. 1976, p. 11-22. 